\title{
The Appropriate Allocation of CEUS in the Diagnostic Algorithm of Liver Lesions: A Debated Issue ${ }^{1}$
}

\section{Bolondi}

\section{Affiliation}

Department of Digestive

Diseases and Internal Medicine, University of Bologna, Italy

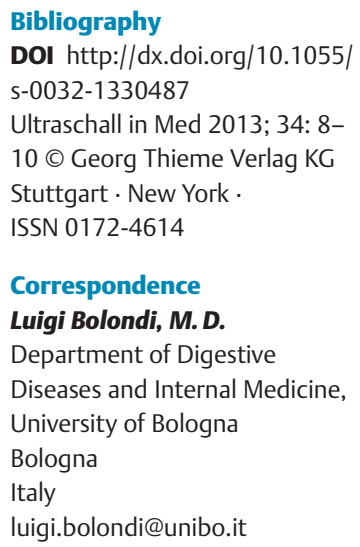

The introduction of a new diagnostic tool into the clinical practice has always been a matter of discussion in the medical literature, both by clinicians and health care managers. There is generally a first phase characterized by enthusiasm and optimism of the authors performing and proposing the new technique, who generally report convincing results that appear significantly better than those achieved by previous techniques in the same field. The counterpart of this optimism is frequently the skepticism of the majority of clinicians not directly involved in the technique. The subsequent phase, often occurring many years later, is characterized by a more balanced evaluation leading, finally, the scientific societies to produce clinical guidelines, in which a quite general agreement about the advantages and limitations of the technique and its diagnostic accuracy has been reached.

This progressive adjustment seems to be particularly complicated and much debated for new advances in ultrasound technology, starting from the introduction of real-time and pulsed and color Doppler and continuing to the recent advent of ultrasound contrast agents (UCAs). It is my opinion that, as far as the ultrasound technology is concerned and, in particular, the use of UCAs, the skepticism and the lack of confidence reported in the literature by nonultrasound-experts, both radiologists and clinicians, has been far greater than for any other technique.

Nevertheless, the use of UCAs and the role of contrast-enhanced ultrasound (CEUS) have nowadays been largely accepted in clinical practice, and the technique has been implemented in most centers for more than a decade. However, CEUS is still waiting for a definite allocation in the diagnostic armamentarium, particularly with respect to computed tomography (CT) scans and magnetic resonance imaging (MRI). Experts in ultrasonography have the feeling that this technique truly represents a great revolution, especially for the diagnosis of liver diseases, allowing in a very quick, simple, and economic way, to provide findings similar to or sometime superior to those obtained by the more sophisticated and expensive CT scans and MRIs, whose availability is and will remain limited. This revolution, however, has not yet been universally understood and acknowledged and, as a consequence, the application of CEUS worldwide is extremely variable.

It is worth remarking that the advantages of CEUS have already been extensively reported in the literature of the past decade. Among them, its costeffectiveness has to be emphasized, especially now, when economic resources face the increased medical and health bills. An imaging technique (CEUS) with a diagnostic accuracy for the characterization of hepatic tumors of about $90 \%$ to $92 \%$ $[19,23]$, comparable to the rates of CT and MRI, that costs less to the national health system, also considering cases of inconclusive diagnoses where another imaging technique is required $[10,16-18$, $21]$ is now available for clinicians. Therefore, why to date has its use not been strongly encouraged by all scientific societies and health care providers and accepted by the whole clinical community? Many reasons underlying the variability of use of CEUS may be searched and analyzed. From the clinical standpoint they include the fact that UCAs were introduced into the clinical practice significantly later than CT and MRI contrast agents, and therefore their contribution has not been immediately appreciated because similar results, with more readable images, could already be obtained by those techniques. Furthermore, most clinicians are resistant to accept changes in their own practice, and they still consider CEUS as something that has been artificially added to CT and MRI, but actually is unable to replace them. Other important points, which have always negatively affected the judgment of any ultrasound technique, are the defect in the standardization, the operator dependence, the variability of results related to the physical characteristics of any individual patient and, last but not least, the difficulty in the interpretation of images by nonexperts, in comparison to CT and MRI. All these points are again a problem for CEUS. These issues play an important role when clinicians without personal experience in ultrasound are included in the panel of experts requested to produce practical guidelines in which recommendations are based on data coming from the literature and expert opinions: for the latter point, personal expertise in ultrasound is more important than it is in any other diagnostic technique. Added to these issues, there is also the problem of registration for UCAs, which has not yet been achieved in some important countries such as the United States and which is difficult to understand based on a strictly medical evaluation of

\footnotetext{
${ }^{1}$ Guidelines and good clinical practice recommendations for contrast-enhanced ultrasound in the liver: update 2012: A World Federation for Ultrasound in Medicine and Biology (WFUMB)-European Federation of Societies for Ultrasound in Medicine \& Biology (EFSUMB) initiative in cooperation with representatives of the Asian Federation of Societies for Ultrasound in Medicine (AFSUMB), the American Institute of Ultrasound in Medicine (AIUM), the American Society of Ultrasound in Medicine (ASUM), and the International Contrast Ultrasound Society (ICUS).
} 
published data. There are many arguments suggesting that the reasons for the lack of registration may probably be economical and/or political, or even simply based on industrial strategies. The latter point may also account for the registration of different UCAs in Europe and in Japan.

Despite these problems, however, the diffusion of CEUS for the diagnosis of liver diseases has progressively increased worldwide, and this poses a question of defining the fields of correct application and of the position of the technique in the diagnostic algorithm.

Many practical guidelines have indeed been produced in recent years regarding the diagnosis of hepatocellular carcinoma (HCC), which is one of the major fields of possible application of CEUS in liver diseases, and it is surprising to remark how the role acknowledged to CEUS is different in the various guidelines. The most striking example is provided by the guidelines produced by hepatologic societies, such as the American Association for the Study of Liver Diseases (AASLD), the European Association for the Study of the Liver (EASL), and the European Organisation for Research and Treatment of Cancer (EORTC), for the diagnosis of HCC in liver cirrhosis. Although the first EASL document [4] included Doppler ultrasound (US) among the techniques able to provide the typical vascular pattern of HCC, and subsequently the first version of AASLD guidelines [2] replaced Doppler US with CEUS, this technique was unexpectedly deleted in the recent updated version of both AASLD and EASL guidelines [3, 9]. Reasons for this change have been officially based on the difficulty of CEUS in distinguishing HCC from intrahepatic colangiocellular carcinoma and emerged from a single paper produced by 1 of the authors of the guidelines [24], but to the majority of clinicians they remain unclear. The lack of registration of CEUS in the United States and, therefore, the problem in recommending a technique that is not available in clinical practice may contribute to this position. What is difficult to understand is why EASL has passively accepted this position despite UCAs' (SonoVue, Bracco, Milan Italy) having been registered in all European countries and their being widely used everywhere. As opposed to the American and European hepatologic societies, the Asian societies (Asian Pacific Association for the Study of the Liver [APASL]) and the Japan Society of Hepatology (JSH) produced their own guidelines for the diagnosis of HCC $[13,15]$, which include CEUS as a technique able to display the typical vascular findings in HCC, and it is included in the recommended diagnostic algorithm. It is worth remarking how the UCA used in Japan (Sonazoid, Daiichi Pharmaceutical/GE Healthcare, Chalfont St. Giles, UK) is different from that used in Europe (SonoVue), but this does not explain the differing positions in the evaluation of the role of CEUS. Looking at these guidelines, an opposite evaluation of the role of CEUS seems to exist between Eastern and Western experts.

My interpretation is that all documents providing recommendations reflect the minds of the authors and their differing clinical and ultrasound expertise. The situation is different in Eastern countries and especially in Japan, where hepatologists are personally performing US. Another point to be underlined is the following: when a panel of experts includes all specialists and clinicians coming from both Eastern and Western countries (as occurred in the first AASLD guidelines; [2]), a more general agreement can be reached concerning the role of CEUS.

This complicated situation has lead the European Federation of Societies for Ultrasound in Medicine \& Biology (EFSUMB), since 2004 , to produce guidelines $[1,6]$ for the clinical use of UCA, and now the World Federation for Ultrasound in Medicine and Biolo- gy (WFUMB) and EFSUMB have updated the previous EFSUMB guidelines, providing the document that is published in this issue of this journal. The work has been conducted in cooperation with representatives of the Asian Federation of Societies for Ultrasound in Medicine (AFSUMB), the American Institute of Ultrasound in Medicine (AIUM), the American Society of Ultrasound in Medicine (ASUM), and the International Contrast Ultrasound Society (ICUS) and includes a large panel of experts that is comprehensive of all clinical, radiologic, and ultrasound experts, thus offering the possibility of full understanding and use of the potential of CEUS, avoiding the bias caused by lack of specific clinical ultrasound expertise present in the updated AASLD [3] and EASL [9] clinical guidelines. This bias led to the underscoring of many current advantages of CEUS, such as: (1) the possibility of monitoring continuously the perfusion of UCA after administration by means of real-time imaging and then of appreciating any rapid hyperenhancement followed by early washout, which are not appreciable with the use of other contrast imaging techniques; (2) the opportunity to define immediately a diagnosis after detection of a liver lesion during US surveillance in cirrhosis, with obvious positive effects in terms of speeding the diagnostic workup and the clinical and psychologic impacts on patients; (3) the possibility of immediately monitoring the effects of any ablative treatment and eventually of completing it on site; (4) the absence of ionizing radiations; (5) the demonstrated cost-effectiveness; and finally (5) the good diagnostic accuracy, similar in most instances to that of $\mathrm{CT}$.

Taking into account all these positive issues and extensively analyzing data from the literature, the updated WFUMB-EFSUMB guidelines now provide a correct allocation of CEUS in each clinical setting in the field of liver diseases, and they represent the best available contribution to the correct use of this technique in the clinical practice. The importance and the validity of these guidelines are further emphasized by the very recent document by the British National Institute for Health and Clinical Excellence ([8]; NICE diagnostic guidance number 5, issued August 2012, www.nice.org.uk/dg5), which endorsed CEUS with SonoVue as the first-line diagnostic assessment in patients with incidentally detected liver lesions and also in patients with nodules in liver cirrhosis (different from the AASLD and EASL guidelines) [3, 9]. NICE is generally very strict in selecting contributions from the literature and in recognizing the validity of any diagnostic tool or drug, so the EFSUMB should be proud that its name is cited 7 times in the full NICE leaflet, mainly in relation to its guidelines. There is a final issue that I would like to emphasize: the future perspectives of this technique. In my opinion, one of the greatest possibilities of UCAs is the strong potential of developing many new compounds with differing vascular and tissue diffusion. Long-lasting liver-phase UCAs, such as BR14 [12] and the abovementioned Sonazoid [11], represent 2 of the main recent technical advances. Furthermore, molecular imaging using targeted UCAs has been already studied in animal models, with promising results for the determination of angiogenesis and with potential translation into clinics, being at last nonimmunogenic $[5,20]$. Innovations in this field also include quantification software, aimed at overcoming subjectivity in evaluating the intensity of enhancement regarding both the characterization of hepatic lesions and the assessment of response to treatment [7, 14, 22].

All these perspectives have been only preliminarily and very partially explored, but the very rapid development of this field induces us to believe that the WFUMB-EFSUMB guidelines for the use of CEUS will need further update in the near future. 


\section{References}

1 Albrecht T, Blomley M, Bolondi L et al. Guidelines for the use of contrast agents in ultrasound. Ultraschall in Med 2004; 25: 249-256

2 Bruix J, Sherman M. Practice Guidelines Committee, American Association for the Study of Liver Diseases Management of hepatocellular carcinoma. Hepatology 2005; 42: $1208-1236$

3 Bruix J, Sherman M, American Association for the Study of Liver Diseases. Management of hepatocellular carcinoma: An update. Hepatology 2011; 53: 1020-1022

4 Bruix J, Sherman M, Llovet JM et al. Clinical management of hepatocellular carcinoma. Conclusions of the Barcelona-2000 EASL conference. European Association for the Study of the Liver. J Hepatol 2001; 35 : $421-430$

5 Bzyl J, Palmowski M, Rix A et al. The high angiogenic activity in very early breast cancer enables reliable imaging with VEGFR2-targeted microbubbles (BR55). Eur Radiol 2012, (in press). DOI: 10.1007/ s00330-012-2594-z

6 Claudon M, Cosgrove D, Albrecht T et al. Guidelines and good clinical recommendations for contrast enhanced ultrasound (CEUS): Update 2008. Ultraschall in Med 2008; 29: 28 - 44

7 Dietrich CF, Averkiou MA, Correas JM et al. An EFSUMB introduction into dynamic contrast-enhanced ultrasound (DCE-US) for quantification of tumour perfusion. Ultraschall in Med 2012; 33: 344-351

8 DG5 SonoVue (sulphur hexafluoride microbubbles): Contrast agent for contrast-enhanced ultrasound imaging of the liver: Full guidance. 2012, Available at www.nice.org.uk/dg5

9 EASL-EORTC clinical practice guidelines: Management of hepatocellular carcinoma. J Hepatol 2012; 56: 908 - 943

10 Faccioli N, D'Onofrio M, Comai A et al. Contrast-enhanced ultrasonography in the characterization of benign focal liver lesions: Activity-based cost analysis. Radiol Med 2007; 112: 810-820

11 Hatanaka K, Kudo M, Minami Y et al. Differential diagnosis of hepatic tumors: Value of contrast-enhanced harmonic sonography using the newly developed contrast agent. Sonazoid. Intervirology 2008; 51: $61-69$

12 Hohmann J, Muller A, Skrok J et al. Detection of hepatocellular carcinoma and liver metastases with BR14: A multicenter phase IIA study. Ultrasound Med Biol 2012; 38: 377-382

13 Kudo M, Izumi N, Kokudo N et al. Management of hepatocellular carcinoma in Japan: Consensus-Based Clinical Practice Guidelines proposed by the Japan Society of Hepatology (JSH) 2010 updated version. Dig Dis 2011; 29: 339-364

14 Lassau N, Koscielny S, Chami L et al. Advanced hepatocellular carcinoma: Early evaluation of response to bevacizumab therapy at dynamic contrast-enhanced US with quantification: Preliminary results. Radiology $2011 ; 258: 291-300$

15 Omata M, Lesmana LA, Tateishi R et al. Asian Pacific Association for the Study of the Liver consensus recommendations on hepatocellular carcinoma. Hepatol Int 2010; 4: 439-474

16 Piscaglia F, Leoni S, Cabibbo G et al. Cost analysis of recall strategies for non-invasive diagnosis of small hepatocellular carcinoma. Dig Liver Dis 2010; 42: 729-734

17 Romanini L, Passamonti M, Aiani L et al. Economic assessment of contrast-enhanced ultrasonography for evaluation of focal liver lesions: A multicentre Italian experience. Eur Radiol 2007; 17: F99-F106

18 Sirli R, Sporea I, Martie A et al. Contrast-enhanced ultrasound in focal liver lesions: A cost-efficiency study. Med Ultrason 2010; 12: 280-285

19 Strobel D, Seitz K, Blank W et al. Contrast-enhanced ultrasound for the characterization of focal liver lesions: Diagnostic accuracy in clinical practice (DEGUM multicenter trial). Ultraschall in Med 2008; 29: 499-505

20 Tardy I, Pochon S, Theraulaz $M$ et al. Ultrasound molecular imaging of VEGFR2 in a rat prostate tumor model using BR55. Invest Radiol 2010; 45: 573-578

21 Tranquart F, Correas JM, Ladam Marcus V et al. Real-time contrast-enhanced ultrasound in the evaluation of focal liver lesions: Diagnostic efficacy and economical issues from a French multicentric study. J Radiol 2009; 90: 109-122

22 Tranquart F, Mercier L, Frinking P et al. Perfusion quantification in contrast-enhanced ultrasound (CEUS): Ready for research projects and routine clinical use. Ultraschall in Med 2012; 33: S31 -S38

23 Trillaud H, Bruel JM, Valette PJ et al. Characterization of focal liver lesions with SonoVue-enhanced sonography: International multicenter-study in comparison to CT and MRI. World J Gastroenterol 2009; 15: $3748-3756$

24 Vilana R, Forner A, Bianchi L et al. Intrahepatic peripheral cholangiocarcinoma in cirrhosis patients may display a vascular pattern similar to hepatocellular carcinoma on contrast-enhanced ultrasound. Hepatology 2010; 51: 2020-2029 